\title{
One Step Quick Detection of Cancer Cell Surface Marker by Integrated NiFe-based Magnetic Biosensing Cell Cultural Chip
}

\author{
Chenchen Bao ${ }^{1, \ddagger}$, Lei Chen ${ }^{1, \ddagger}$, Tao Wang ${ }^{1}, \quad$ Chong Lei $^{1}$, Furong $\operatorname{Tian}^{2}$, Daxiang Cui ${ }^{1, *}, \quad$ Yong Zhou ${ }^{1, *}$
}

(Received 10 July 2013; accepted 05 September 2013; published online 16 September 2013)

\begin{abstract}
RGD peptides has been used to detect cell surface integrin and direct clinical effective therapeutic drug selection. Herein we report that a quick one step detection of cell surface marker that was realized by a specially designed NiFe-based magnetic biosensing cell chip combined with functionalized magnetic nanoparticles. Magnetic nanoparticles with $20-30 \mathrm{~nm}$ in diameter were prepared by coprecipitation and modified with RGD-4C, and the resultant RGD-functionalized magnetic nanoparticles were used for targeting cancer cells cultured on the NiFe-based magnetic biosensing chip and distinguish the amount of cell surface receptor-integrin. Cell lines such as Calu3, Hela, A549, CaFbr, HEK293 and HUVEC exhibiting different integrin expression were chosen as test samples. Calu3, Hela, HEK293 and HUVEC cells were successfully identified. This approach has advantages in the qualitative screening test. Compared with traditional method, it is fast, sensitive, low cost, easy-operative, and needs very little human intervention. The novel method has great potential in applications such as fast clinical cell surface marker detection, and diagnosis of early cancer, and can be easily extended to other biomedical applications based on molecular recognition.
\end{abstract}

Keywords: Magnetic impedance; Cancer cell; Targeted detection; Biosensing system; Cell cultural chip

Citation: Chenchen Bao, Lei Chen, Tao Wang, Chong Lei, Furong Tian, Daxiang Cui and Yong Zhou, "One Step Quick Detection of Cancer Cell Surface Marker by Integrated NiFe-based Magnetic Biosensing Cell Cultural Chip", Nano-Micro Lett. 5(3), 213-222 (2013). http://dx.doi.org/10.5101/nml.v5i3.p213-222

\section{Introduction}

There are many methods for detection of biomolecular targets in the field of life science. Diagnosis of disease is usually carried out by isolation and cultivation of microorganisms, which may take several days and demand special technical skills and complex, expensive equipment. In such cases, biosensors [1], which can incorporate a biological or biological-derived sensing element that is associated with or interacted within a physicochemical transducer, are introduced into biological analytical systems for its properties of high sensitivity and specificity [2], large scale, high speed, low cost and minimum human intervention [3], etc. Among biosensors, magnetic biosensors gained their popularities because firstly, the magnetic background in biomolecular samples are usually insignificant; secondly, they are relatively inexpensive and easily to be made biocompatible [4]; thirdly, magnetic property is very stable over time; Finally, many applications can be made based on the stray magnetic fields from the particles and the external fields [5]. They are highly sensitive among magnetic biosensors and also portable [6]. Moreover, they can give a fully electronic readout and possess the potential of large scale detective appli-

\footnotetext{
${ }^{1}$ Key Laboratory for Thin Film and Microfabrication of Ministry of Education, Research Institute of Micro/Nano Science and Technology, Shanghai Jiao Tong University, Shanghai 200240, China

${ }^{2}$ FOCAS Research Institute, Dublin Institute of Technology, Kevin Street, Dublin 8, Ireland

¥These authors contributed equally to this work

*Corresponding authors. E-mail: dxcui@sjtu.edu.cn
} 
cations. Based on previous report, magnetic biosensors can not only be introduced to detect thin ferrofluid layers [7], micro-beads [8] and nanoparticles [9], but also be possible to be used for molecular recognition events, e.g. human papilloma virus (HPV), hepatitis B virus (HBV) and gastric cancer cells [12], or even in a label-free manner via surface morphology or anisotropy changes [13]. One of the tendencies for biosensing chip is low cost and high sensitivity in order to promote a wide range application in hospital and clinical. Micro-electromechanical system (MEMS) technology is an excellent way to mass fabricate magnetic biosensing chip with low cost. Also it is easier to fabricate magnetic sensing element array of biosensing chip in MEMS way to get obvious highly sensitivity of magnetic sensing element [14].

RGD peptides have been extensively investigated as cell adhesion [15] peptide that is recognized by cellsurface receptors, such as integrin known to mediate cell adhesion and proliferation. This adhesion molecule plays a key role in tumor angiogenesis and metastasis, being considerably up-regulated on endothelium during angiogenesis. Therefore, RGD has represented a marker for malignancy [16], and become a popular tool [17] for the targeting of drugs and part of functionalized imaging contrast agents [18-20]. Traditionally, cell surface receptors can be detected by a flow cytometry [21], or fluorescent microscopy, in the method of detect light signal from commercialized fluorescent dyes. However, this process involves in expensive reagents (fluorescent monoclonal antibodies and their isotype controls), expensive equipment (flow cytometer or fluorescent microscopy), long procedures (from cell culture, collection, fixation, staining and detection), and skillful technicians. Though our previous study proved NiFe based cell cultural sensors are possible to be used for cancer cell detection [12], we have not applied it for certain cell surface markers detection among different cell lines, nor have we combined the cell culture and detection process together [12].

In this paper, we propose an integrated NiFe-based magnetic biosensing cell cultural chip and its array that fabricated by MEMS technology to detect cell surface marker integrin $\alpha_{v} \beta_{3}$. This novel detection system possesses a cell culture surface, a highly sensitive biosensor, functionalized superparamagnetic nanoparticles, high specificity, fast and accuracy of cell surface markerintegrin detection.

\section{Materials and methods}

\section{Preparation and characterization of functional- ized magnetic nanoparticles}

Iron (III) chloride of $2.3 \mathrm{~g}$ and Iron (II) sulfate heptahydrate of $2.2 \mathrm{~g}$ were dissolved in deionized water separately before mixed with a total volume of 100
$\mathrm{mL}$. This mixture was vigorously stirred in argon atmosphere at the room temperature for $30 \mathrm{~min}$. Chitosan solution was prepared by dissolving $0.6 \mathrm{~g}$ chitosan in $25 \mathrm{~mL}$ water and mixing with $5 \mathrm{~mL}$ trisodium citrate dehydrate solution $(0.4 \mathrm{~g} / \mathrm{mL}) .15 \mathrm{~mL}$ of Ammonia solution (wt 25\%) was diluted in $35 \mathrm{~mL}$ of water before it was dropped into the flask within $2 \mathrm{~min}$. The flask was soon removed to water bath at the temperature of $70^{\circ} \mathrm{C}$ for 30 min followed by the mix of the chitosan solution. It takes $1 \mathrm{~h}$ more in the water bath before the black suspension was cooled under room temperature. After washed several times with anhydrous alcohol, large particles were removed from the suspension by means of magnetic separation. The $\mathrm{Fe}_{3} \mathrm{O}_{4} @$ chitosan nanoparticles were washed and diluted with water. After washed by $0.01 \mathrm{~mol} / \mathrm{L} 2$-( $N$-morpholino)ethanesulfonic acid (MES) buffer ( $\mathrm{pH}$ 6.3) before activated with 6.5 nmol/L 1-Ethyl-3-(dimethylaminopropyl)carbodiimide hydrochloride (EDC) and $22 \mathrm{nmol} / \mathrm{L} \mathrm{2-(N-morpholino)-}$ ethane sulfonic acid (NHS) MEST buffer, $200 \mathrm{mg}$ of RGD-4C were added to $500 \mu \mathrm{L}$ of $30 \mathrm{mg} / \mathrm{mL}$ prepared nanoparticles and incubated for $2 \mathrm{~h}$ at room temperature. Then the RGD conjugated nanoparticles were purified by magnetic separation and rinsed thrice with PBS buffer.

After dried in vacuum at $-54^{\circ} \mathrm{C}$ for $24 \mathrm{~h}$, the $\mathrm{Fe}_{3} \mathrm{O}_{4} @$ chitosan nanoparticles were characterized by a transmission electron microscopy (TEM, JEM2010/INCA OXFORD) and a field emission scanning electron microscope (Zeiss Ultra 55). X-ray powder diffraction (XRD, D/max-2200/PC) pattern and thermogravimetric analysis (TGA) (PerkinElmer Pyris 1) of the $\mathrm{Fe}_{3} \mathrm{O}_{4} @$ chitosan are also illustrated. Dried $\mathrm{Fe}_{3} \mathrm{O}_{4} @$ chitosan (1-5 mg) was placed in the TGA furnace and the measurement was carried out with a heating rate of $0.5^{\circ} \mathrm{C} / \mathrm{min}$ from 27.42 to $860.48^{\circ} \mathrm{C}$. Infrared spectrum (EQUINOX 55, Bruker Co., Germany) and SEM image are illustrated in the supplementary materials.) The amount of RGD on the nanoparticles was determined by the quantification of RGD solution before and after coupling reaction by BCA protein assay (raw data unshown). The function of the prepared RGD- $\mathrm{Fe}_{3} \mathrm{O}_{4} @$ @hitosan was proved by competitive inhibition experiment.

\section{The fabrication of detective cell cultural chip}

The detective cell cultural chip is consisted of NiFe magnetic sensing element and poly dimethyl siloxane (PDMS) cavity. NiFe thin films in magnetic sensing element array are prepared by RF magnetron sputtering onto 3-inch glass substrates, as shown in Fig. 2(a). The diameter of commercial NiFe target is 5 inch and the purity is $99.999 \%$. Before sputtering, the chamber is evacuated to below $8 \times 10^{-7}$ Torr. A constant magnetic field of about 200 Oe is applied along the transverse direction 
in the film plane during the deposition process in order to induce the transverse anisotropy. The structure of each individual sensing element is $\mathrm{NiFe} / \mathrm{Cu} / \mathrm{NiFe}$ multilayer films. The length of sensing is $5 \mathrm{~mm}$, the width of each line is $500 \mu \mathrm{m}$ and the space between each line is $200 \mu \mathrm{m}$, as shown in Fig. 2(b). The sensing element also have an inner $\mathrm{Cu}$ layer with two extended electrodes and rectangle PDMS cavity, as shown in Fig. 2(c). $\mathrm{SiO}_{2}$ layer is deposited on the surface of sensing element as an insulated and protective layer.

The magnetic effects of sensing element is measured by an impedance analyzer (HP4194A) with two contact terminals, the AC current flows through the sensors with frequency $20 \mathrm{MHz}$ and with the constant current amplitude of $10 \mathrm{~mA}$. An external magnetic field $\mathrm{H}$ of $0-100 \mathrm{Oe}$ is applied to the sensing element. The relative change of magnetic-impedance of sensing element, is calculated from $Z(H)$ curves and is defined as: magnetic-impedance ratio $=100 \% \times$ $\left[Z(H)-Z\left(H_{\max }\right)\right] / Z\left(H_{\max }\right)$, where $Z(H)$ is the magnetoimpedance with $0 \sim 100$ Oe magnetic field and $Z\left(H_{\max }\right)$ is the 100 Oe magnetic field applied, respectively. Normally, magnetic nanoparticles detection based on magnetic-impedance ratio follows closely ideas from Baselt [6] in which the particles and sensors are exposed to external magnetic fields, in the plane of the sensor, which induces a magnetic dipole in the particle aligned to the applied field. The field in the in-plane case is mostly unidirectional and is opposite in sense to the applied field. In this case, the presence or absence of the particle modifies the effective applied field to the sensor, and consequently alters the sensor magnetoimpedance and make the magnetic-impedance ratio is different. The magnetic-impedance ratio change can qualitatively reflect the presence or absence of the magnetic nanoparticles, even can quantitatively index the amount of magnetic nanoparticles contained in biology samples. This is the principle of our experiment method. More detailed information of magneticimpedance ratio property and fabrication method of NiFe sensing element and PDMS cavity in our work can be found elsewhere $[12,14]$.

\section{Analysis of RGD-Fe ${ }_{3} \mathrm{O}_{4} @$ chitosan uptake in hu- man gastric cancer cells}

Cell culture

MGC-803 Cells were cultured in complete cell culture medium composed of RPMI 1640 with L-Glutamine (Invitrogen, Germany) supplemented with 10\% (v/v) fetal Bovine Serum (Sigma Aldrich, Germany) and 1\% (v/v) 10,000 U/mL Penicillin and 10,000 U/mL Streptomycin (Invitrogen, Germany). Cells were maintained under standard cell culture conditions $\left(5 \% \mathrm{CO}_{2}, 95 \%\right.$ humidity and at $37^{\circ} \mathrm{C}$ ) in Thermo incubators and passed 2 or 3 days.
MGC-803 cells were incubated on 6-well plates with $0.35 \mu \mathrm{mol} / \mathrm{mL}$ plain $\mathrm{Fe}_{3} \mathrm{O}_{4} @$ chitosan or RGD modified $\mathrm{Fe}_{3} \mathrm{O}_{4} @$ chitosan for 5 different time intervals: $10 \mathrm{~min}$, $30 \mathrm{~min}, 1 \mathrm{~h}, 3 \mathrm{~h}$ and $5 \mathrm{~h}$. Free RGD-4C and RGD$\mathrm{Fe}_{3} \mathrm{O}_{4} @$ chitosan was mixed in a ratio of 10,000:1 before added in to the competition group. After incubation, the culture medium was removed.

For Prussian blue staining, MGC803s were washed twice with PBS buffer and subsequently fixed with cold methanol and acetone. The fixed cells were incubated with $10 \%$ potassium ferrocyanide for $5 \mathrm{~min}$ and $10 \%$ potassium ferrocyanide in $20 \%$ hydrochloric acid for 30 min, and counterstained with nuclear fast red.

\section{The co-culture of cell chip and cancer cells and the biocompatibility test}

Before put in to a $10 \mathrm{~cm}$ cell culture dish, the produced cell chip was sterilized in autoclave as regular process $\left(121^{\circ} \mathrm{C}\right.$ for $\left.30 \mathrm{~min}\right)$. Cells were incubated $6 \mathrm{~h}$, $12 \mathrm{~h}, 24 \mathrm{~h}, 36 \mathrm{~h}, 48 \mathrm{~h}$ in a $5 \% \mathrm{CO}_{2}, 95 \%$ air humidified incubator at $37^{\circ} \mathrm{C}$. After incubation, cells from each condition were digested with $0.25 \%$ trypsin before MTS assay and LDH leakage tests. The standard procedures can be found elsewhere [22].

\section{The detection of cell surface integrin by flow cy- tometry method}

Cell surface protein expression of integrin was determined by flow cytometry (FCM) analysis. Briefly, cells were washed with Phosphate Buffered Saline (PBS) and incubated for 10 minutes with $30 \mathrm{mmol} / \mathrm{L}$ EDPA (Sigma Aldrich) in PBS at $37^{\circ} \mathrm{C}$. Detached cells were scratched, collected and blocked (with 1\% BSA on ice) for one hour, adjust to $1 \times 10^{5}$ cells per $100 \mu \mathrm{L}$ in PBS/1\% Bovine serum albumin (BSA), and successively stained in darkness for 30 minutes at $4^{\circ} \mathrm{C}$ with FITC-mouse IgG1 $\kappa$ isotype control (for control group) or with FITC-Mouse anti-human CD51/61 (BD Biosciences) (for test group). Analysis of 10,000 events was performed by using a BD FACSDiva flow cytometer (Becton Dickinson, Heidelberg, Germany) and BD FACSDiva v6 software. Cells were initially gated on the basis of forward and side scatter characteristics.

\section{Cell detection}

Several typical epithelial cell lines or carcinoma associated cell lines were chosen for the tests. They are human lung carcinoma cells A549, human lung epithelial adenocarcinoma cells Calu-3, human epithelial cells HEK293, human epithelial cervix adenocarcinoma cells Hela, Human umbilical vein endothelial cells HUVEC and carcinoma associated lung fibroblasts CaFbr. $1 \times 10^{7}$ cells were firstly seeded and cultured on the chip for $2 \mathrm{~h}$ before the complete medium was 
removed. Then RGD-Fe $\mathrm{O}_{4} @$ @hitosan nanoparticles or $\mathrm{Fe}_{3} \mathrm{O}_{4} @$ chitosan nanoparticles were add into the dish with an iron concentration of $0.35 \mu \mathrm{mol} / \mathrm{mL}$ for $1 \mathrm{~h}$ followed by rigorous rinse by PBS.

15 types of samples are prepared for the detection: (1) Cells without incubation with any nanoparticles. (The result of co-culture with different cell lines at different ratios was checked before the following steps. Trace amounts of iron in $1 \times 10^{5}$ to $1 \times 10^{7}$ cells for the determination has no interference with the giant magnetoimpedance ratio.); (2)-(6), several cell lines: HUVEC, A549, Hela, Calu3, CaFbr, and HEK293 incubated with $\mathrm{Fe}_{3} \mathrm{O}_{4} @$ chitosan; (7)-(13), all of the chosen cell lines incubated with RGD- $\mathrm{Fe}_{3} \mathrm{O}_{4} @$ @ chitosan respectively. For all the groups, $1 \times 10^{6}$ cells were seeded for the detection. Then, their difference can be determined by the difference of magnetic-impedance property among their corresponding NiFe-based cell chip. Finally, magnetoimpedance of the chip was measured by an impedance analyzer with the extension cable and test fixture connected to the edge of the chip.

We carried out the detection of each sample in tenfold manner and the results are counted as magneticimpedance response of sample group minus its background group. Standard deviation (SD) range of magnetic-impedance response is calculated for each sample.

\section{Data analysis}

The difference from cell surface integrin was calculated as:
Giant magnetoimpedance for cell A = Giant magnetoimpedance of cell A incubated with RGD modified $\mathrm{Fe}_{3} \mathrm{O}_{4} @$ @itosan - Giant magnetoimpedance of cell A incubated with plain $\mathrm{Fe}_{3} \mathrm{O}_{4} @$ chitosan

All data are presented in this paper as mean result \pm S.E. Statistical differences were evaluated using the $t$-test and considered significance at $P<0.05$ level.

\section{Results and discussion}

\section{Characterization of targeted particles}

As illustrated in Fig. 1(a), the mean size of the particles is $20 \mathrm{~nm}$ by TEM. The XRD pattern of $\mathrm{Fe}_{3} \mathrm{O}_{4} @$ chitosan in Fig. 1(b) shows a series of characteristic peaks of $\mathrm{Fe}_{3} \mathrm{O}_{4}$ : 220, 311, 199, 511 and 440. Meanwhile, in thermogram test of $\mathrm{Fe}_{3} \mathrm{O}_{4} @$ chitosan, Fig. 1(c) demonstrated two stages of weight loss. The first one is due to the evaporation of water molecules and the other one can be ascribed to the decomposition of chitosan. These showed that chitosan was successfully coated on the surface of iron oxide particles. Since the nanoscale magnetic nanoparticles possess superior physical and chemical properties, it can be applied in many biomedical processes. For example, the detection noninvasive detection of HBV surface antibodies [23], and gene delivery system [24]. Iron based magnetic nanoparticles can also be produce by gram-scale synthesis [25-27], thus the clinical application can be promising for its low cost and homogeneity.
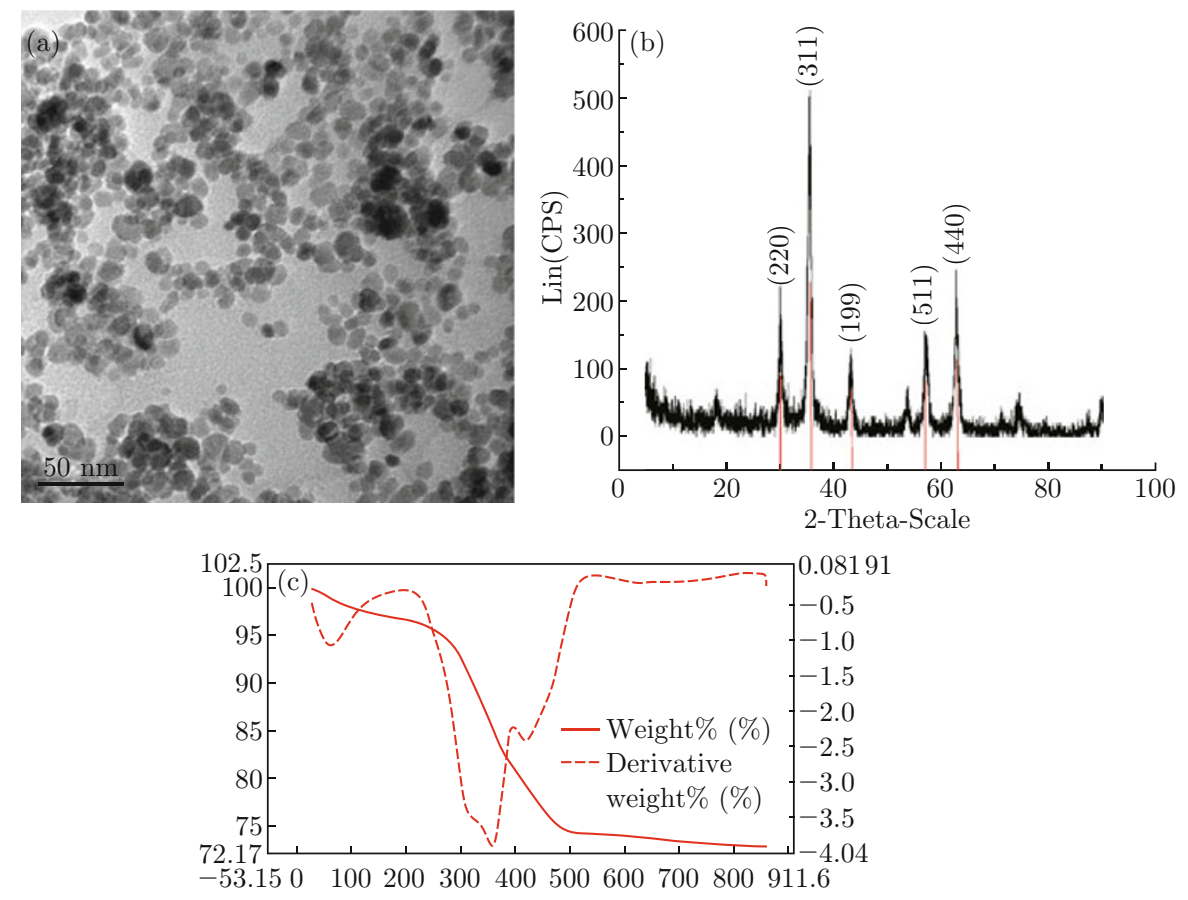

Fig. 1 (a) TEM image of $\mathrm{Fe}_{3} \mathrm{O}_{4} @$ chitosan particles; (b) The XRD pattern of $\mathrm{Fe}_{3} \mathrm{O}_{4} @$ chitosan; (c) The weight loss curves of $\mathrm{Fe}_{3} \mathrm{O}_{4} @$ chitosan. 
Characterization of NiFe-based megnatic biosensing cell cultural chip

As shown in Fig. 2(a), NiFe-based detection cell chip array are fabricated on 3-inch glass substrate. For the magnetic-impedance property of this chip array, more detailed information is illustrated in supplementary materials Fig. S3-S5. It is also possible that NiFe-based cell cultural chip on 3-inch substrate can be cut into single test elements for different biological samples and thus significantly lower the test cost; Moreover, test elements can be constructed into different combinations of test arrays and these test arrays can significantly in- crease the test sensitivity than a single unit. Figure 2 (c) shows the detection area that consists of NiFebased sensing element and PDMS cavity. We sputtered $\mathrm{SiO}_{2}$ layer on the surface of sensing element in order to prevent the directly contact of cell sample and the metal material of sensing element. Considering the tendency of the integration of chips, it is possible to combine integrated circuit (IC) test circuit and sensing element. Due to the compatibility of MEMS and Complementary metal-oxide-semiconductor (CMOS) technology, we can produce independent testing system-ona-chip with miniaturization, high sensitivity and low cost.
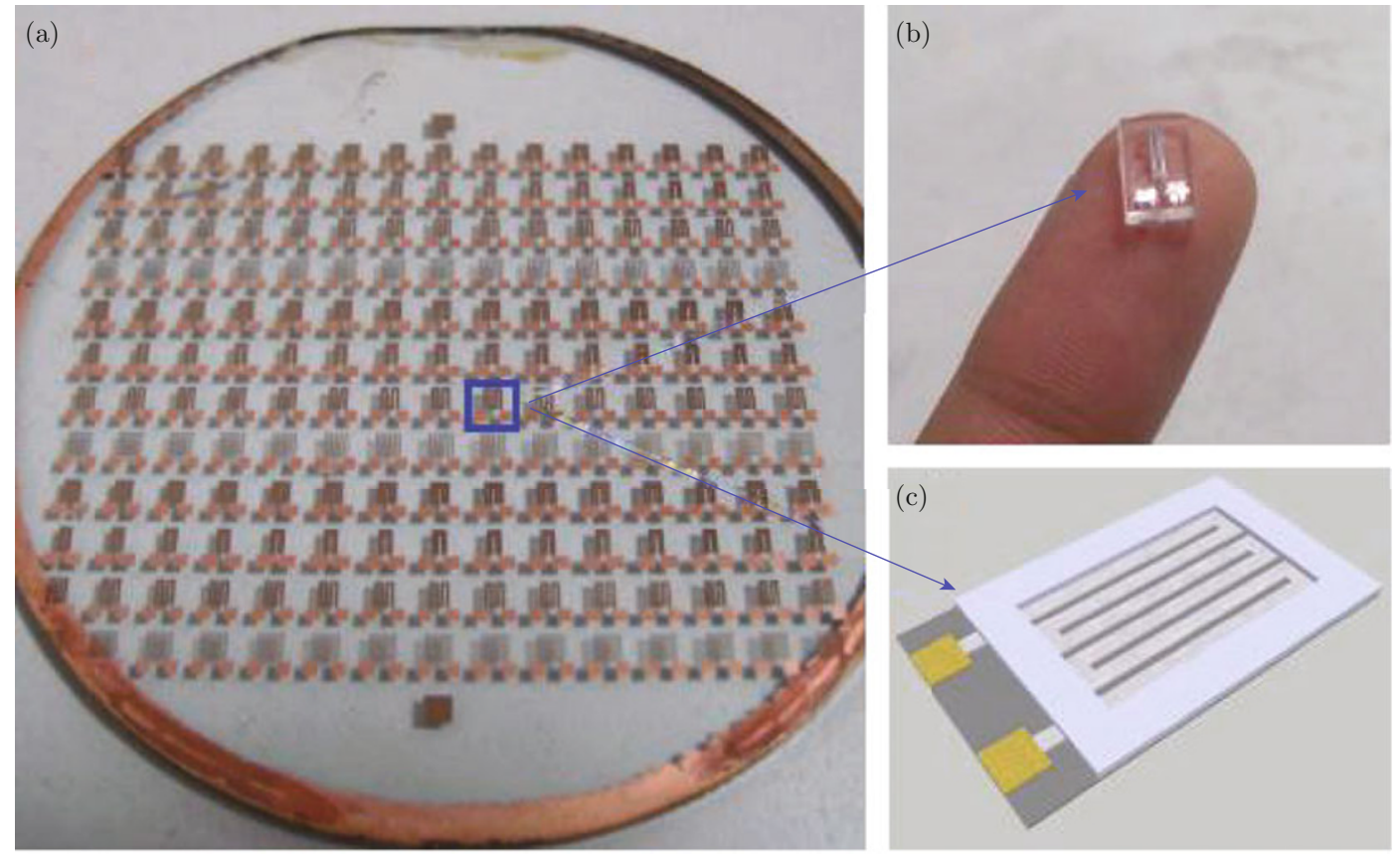

Fig. 2 (a) The photograph of prepared co-culture NiFe-based detection cell chip; (b) Partial magnification of the NiFe-based sensing element; (c) scheme image of NiFe-based sensing element.

\section{Competitive experiment for the functionalized nanoparticles}

The uptake of $\mathrm{Fe}_{3} \mathrm{O}_{4} @$ chitosan, RGD- $\mathrm{Fe}_{3} \mathrm{O}_{4} @$ chitosan and RGD- $\mathrm{Fe}_{3} \mathrm{O}_{4} @$ chitosan plus free RGD after blocking the target by addition of free RGD peptides was assessed histologically using Prussian blue staining [28] (Fig. 3). After $10 \mathrm{~min}$, a strong uptake of RGD$\mathrm{Fe}_{3} \mathrm{O}_{4} @$ chitosan was observed, whereas the uptake for plain $\mathrm{Fe}_{3} \mathrm{O}_{4} @$ chitosan was less significant. Blocking the $\alpha_{v} \beta_{3}$ integrin receptor with free RGD effectively reduced the amount of blue granules in the cytoplasm of MGC-803s, which indicates that the accumulation of the particles was specifically mediated by its integrin binding. After 3-hour incubation, however, the differences between all kinds of nanoparticles used were less prominent. This phenomenon may be due to the extension of time as the dominant factor.

\section{Cancer cell incubation with the NiFe-based cell cultural chip}

The surface of the cell chip was modified with silicon dioxide. The cell morphology is shown as Fig. 4(a). Adherent cells MGC-803 can maintain their morphology on the chip surface. We harvest cultured cells on 6 h, $12 \mathrm{~h}, 24 \mathrm{~h}, 36 \mathrm{~h}$ and $48 \mathrm{~h}$ respectively for cytotoxicity studies. Assays were designed to measure two markers to account for the number of live cells (MTS assay) and dead cells (LDH leakage assay). Cell viability decreased slightly from $100 \%$ to $86 \%$ (Fig. 4 (b)) from 6 h to 48 h. Similarly, in the LDH assay, untreated control cells were defined as baseline (0\%). Only $7 \%$ leakage was observed from cancer cells after $48 \mathrm{~h}$ co-culture with cell chips and only as low as $4 \%$ leakage after $6 \mathrm{~h}$. Therefore, the biocompatibility is good to maintain cancer cells and lasts long enough for the test. 


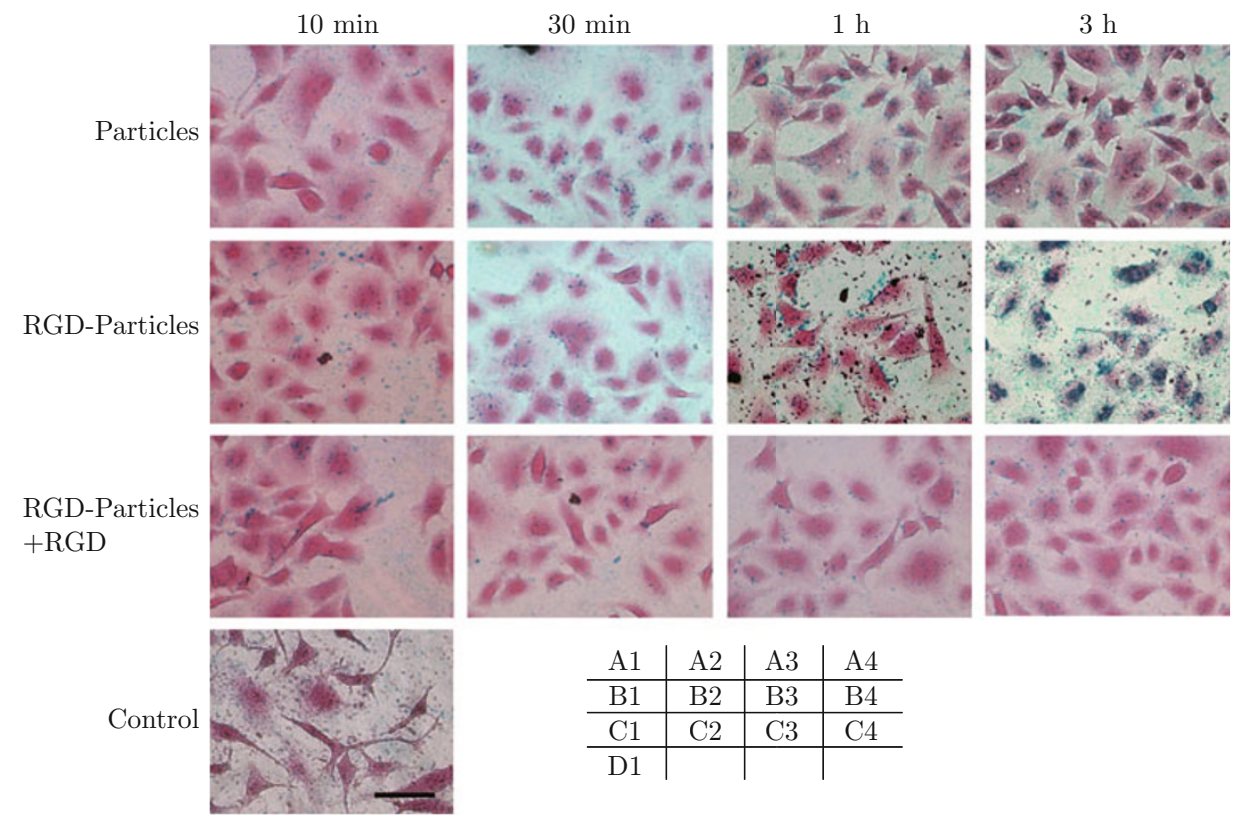

Fig. 3 Prussian blue-stained and nucleus fast red-counterstained MGC-803 incubated with $\mathrm{Fe}_{3} \mathrm{O}_{4} @$ chitosan (A1-A4), RGD$\mathrm{Fe}_{3} \mathrm{O}_{4} @$ chitosan (B1-B4), and RGD- $\mathrm{Fe}_{3} \mathrm{O}_{4} @$ chitosan+RGD (competition group, C1-C4) at an iron concentration of 0.35 $\mu \mathrm{mol} / \mathrm{mL}$ of growth medium for different time intervals (10 min, $30 \mathrm{~min}, 1 \mathrm{~h}$ and $3 \mathrm{~h}$ ). Control cells that were not incubated with any nanoparticles are shown in D1. For all conditions, particle uptake increased significantly for longer incubation time. Higher uptake of RGD- $\mathrm{Fe}_{3} \mathrm{O}_{4} @$ chitosan (blue granules) compared with plain $\mathrm{Fe}_{3} \mathrm{O}_{4} @$ chitosan competition group is clearly indicated. (Bar: $10 \mu \mathrm{m}$ )

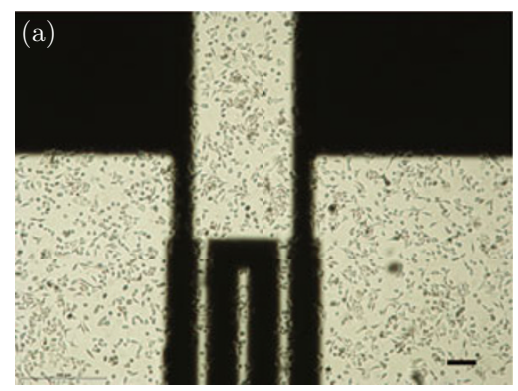

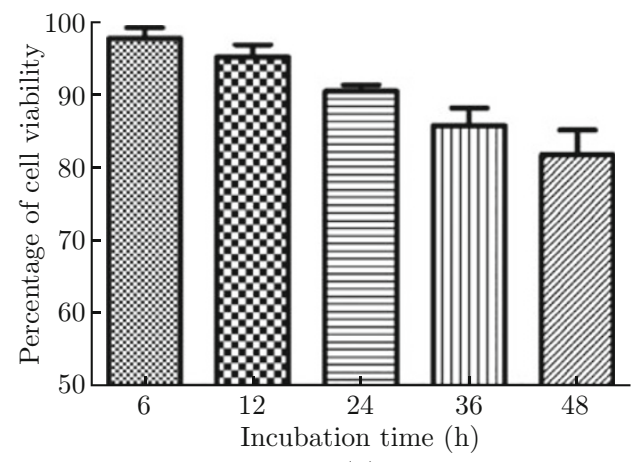

(b)

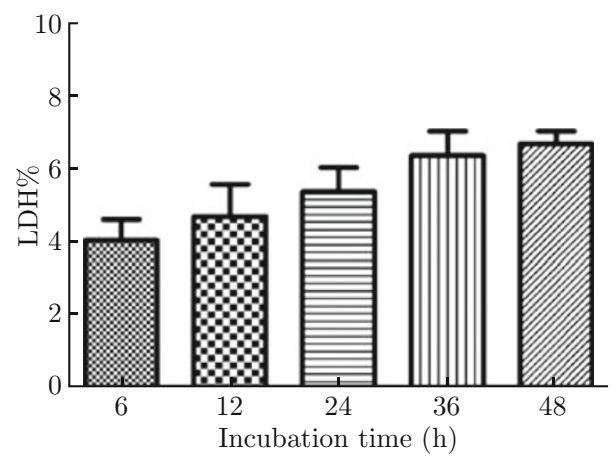

(c)

Fig. 4 (a) Optical microscope images of MGC-803 cell growth on the prepared co-culture NiFe-based cell chip. (Bar: 500 $\mu \mathrm{m})$; (b) Cell viability test for co-cultured MGC-803 cells on the surface of the NiFe-based cell chip $(6 \mathrm{~h}-48 \mathrm{~h})$. (c) Cell membrane leakage at various time intervals $(6 \mathrm{~h}-48 \mathrm{~h})$.

\section{Flow cytometry test on cell surface target}

RGD-surface-modified nanoparticles have been extensively used to deliver therapeutic genes, confirming the involvement of integrin in transfection [29]. In order to confirm different expression of integrin of the chosen cell lines, FCM analysis was performed in HUVEC, A549, Hela, CanFbr, Calu3 and HEK293 cells. 
RGD integrin ligand is well-known for its capability to bind to the integrin $\alpha_{v} \beta_{3}$ receptor family, involved in a wide range of cell extracellular matrix and cell-cell interactions. FCM analysis (Fig. 5) revealed highest levels of the RGD motif binding integrin $\alpha_{v} \beta_{3}$ expressed on HEVECs (84.5\%), compared to CanFbr (12.5\%), A549 (12.0\%), Hela (6.6\%), Calu3 (3.7\%) and HEK293 $(14.0 \%)$ cells. After the excitation by the lasers, the fluorochromes can be detected by detectors as light signal. FCM method can observe cell events in highthroughput manner and it has become a standard detection for decades. However, FCM test may have some disadvantages for large-scale usage. Firstly, the price for a flow cytometer is around 100,000 to 400,000 US dollars, and a single test may cost 90 US dollars per hour. Also, a flow cytometer requires appropriate temperature, humidity and environment for maintenance. It also takes time to pre-warming the system before the test. This is the main reason why FCM method cannot be universal for small clinics and rural areas. Secondly, it takes trained operators, and the observation is subjective for gating. Thirdly, fluorescent dyes suffer from bleaching and quenching and require the prepared samples only kept in coldness and darkness for short time. Therefore, FCM method is more suitable for precise quantitative analysis for small samples, but has its drawbacks for large-scale fast qualitative comparisons.

\section{The detection of targeted tumor cells on cell chip}

Each type of cell line was divided into two groups: sample group (incubated with $\mathrm{RGD}-\mathrm{Fe}_{3} \mathrm{O}_{4} @$ chitosan) and background group (the counterparts incubated with $\mathrm{Fe}_{3} \mathrm{O}_{4} @$ @chitosan ). We have selected a field of 15 and 20 Oe for comparison because in this field range, where the differences are most obvious (Fig. 6(a)). If there is no intersection between two intervals, it can be considered that the two samples have significant difference. If there is overlapping part between SD ranges of two samples, the identification of the two samples is recognized as failed. FCM analysis showed that the expressions of $\alpha_{v} \beta_{3}$ in those cells such as Calu3, Hela, HEK293 and HUVEC are 3.7\%, 6.6\%, $14 \%$ and $84.5 \%$, respectively (Fig. 5). As shown in Fig. 6(b), those samples were successfully identified. However, there is obvious intersection part of SD range between A549 and CaFbr. Therefore, we cannot say that our chip is capable to identify them. Interestingly, in Fig. 5, the quantitative result from flow cytometry also shows the integrin expression of the $\mathrm{A} 549$ and CaFbr are also very close $(12 \%$ and $12.5 \%$, respectively). This finding

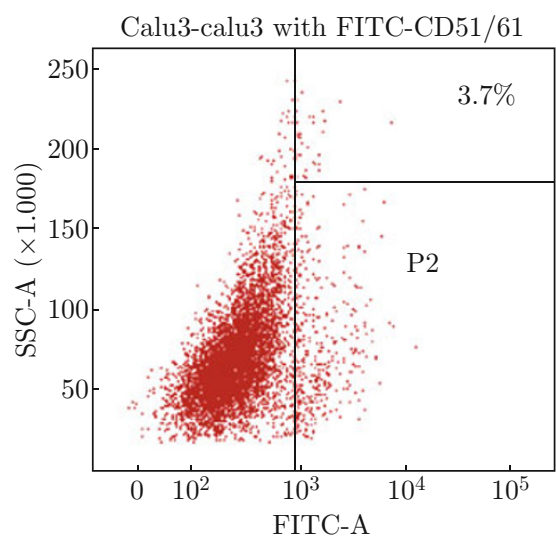

A549-A549 with FITC-CD51/61

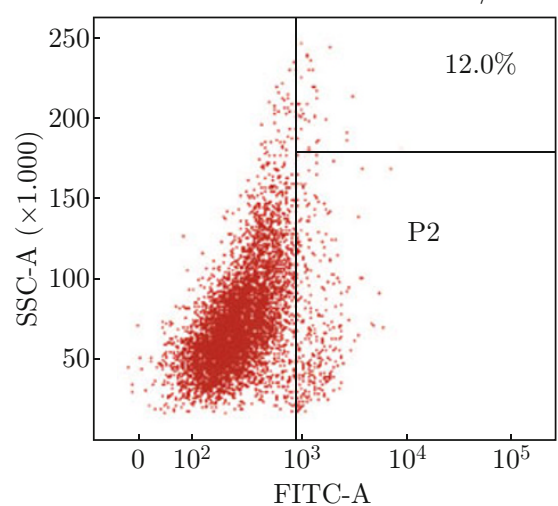

Hela-hela with FITC-CD51/61

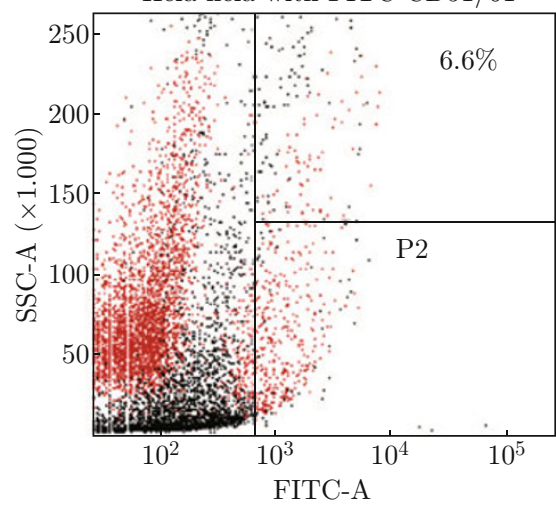

CaFbr-CaFbr with FITC-CD51/61

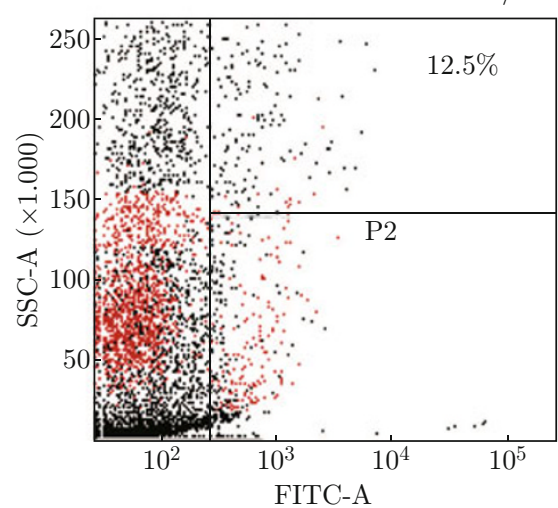

HUVEC-HUVEC with FITC-CD51/61

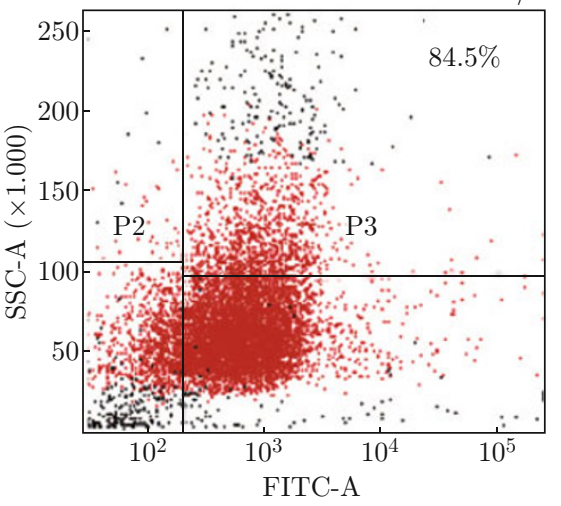

HEK293-HEK293 with FITC-CD51/61

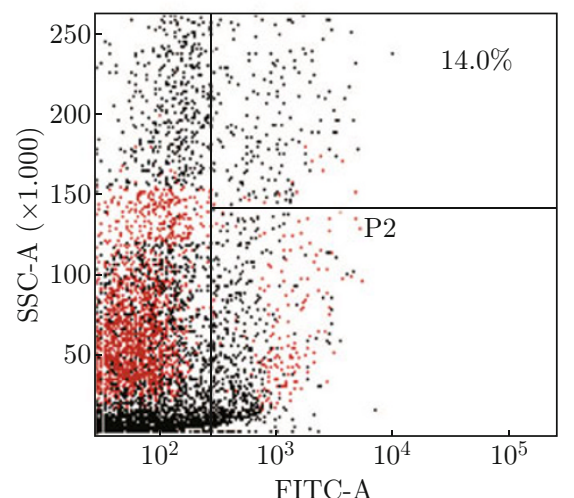

Fig. 5 Flow cytometry ananlysis of the surface receptor $\alpha \mathrm{v} \beta 3$ integrin of the chosen cell lines. 10,000 events were recorded for each cell line. After stained by FITC-CD51/61, the amounts of integrin of different cell lines are Calu-3 3.7\%, Hela 6.6\%, HUVEC 84.5\%, A549 12\%, CaFbr, 12.5\%, HEK293 14\%. 


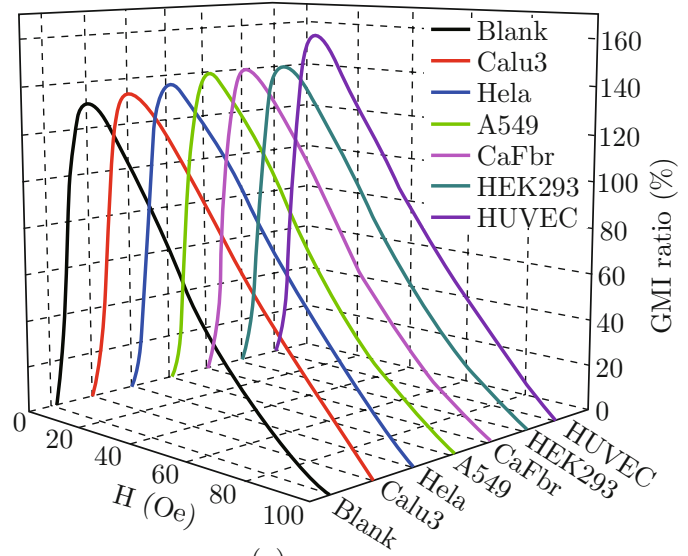

(a)

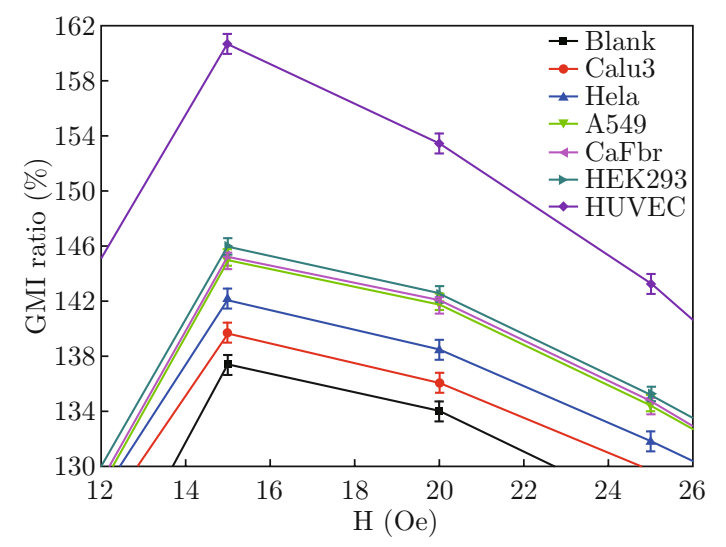

(b)

Fig. 6 (a) Magnetic-impedance response of seven different samples; (b) Standard deviation analysis of 10 repeated test results of seven different samples. The difference between HUVEC and the rest of the test cell lines is significant.

indicated the detection limit of current system. For the samples whose cell surface target expression as close as less than $1 \%$, the system cannot detect significant difference.

There are several factors that may affect the signal outputs from our chip. Firstly, the amount of cells used in the detection is counted. $1 \times 10^{6}$ cells for all the samples were seeded before each test in our experiment. Secondly, the interference from the background of different cell lines was excluded by dividing each cell type into two equal groups- adding $\mathrm{Fe}_{3} \mathrm{O}_{4} @$ chitosan or RGD$\mathrm{Fe}_{3} \mathrm{O}_{4} @$ chitosan nanoparticles. Thirdly, the impedance analyzer was kept in a separate region to prevent magnetic shield.

Cell to cell and cell to matrix interactions are involved in many physiological process including cell differentiation, embryogenesis [30], hemostasis [31], wound healing [32] and immune response [33]. They are also fundamental to tumor invasion and formation of metastasis as well as tumor-induced angiogenesis [29]. Our system can provide information about the receptor status of the tumor and enable specific therapeutic planning. The produced cell cultural chip is easier, cheaper and faster for clinical usage. It serves us as a good alternative with the potentials of large-scale, robustness and repeated usage.

For large-scale qualitative test, our system has obvious advantages. Firstly, there are numerous applications of magnetic nanoparticles functionalized with RGD peptide or monoclonal antibodies or their fragments; for example, our system can be more widely used for biomedical detection. Secondly, very little human intervention is needed during the test, unlike FCM test which requires special trained personnel. Thirdly, compared with quantitative test such like FCM method or fluorescent microscopy, this method is much cheaper. The detector price is far lower than flow cytometry (around $2.5 \%$ to $10 \%$ of the price), and the system can be made into the portable device in near future. For the frequency requirement of our test, it only need to fix one working frequency and we can use some commercial IC chip to instead impedance analyzer-HP4194A. The IC chip is very small and very low price compare with flow cytometry. Also, the NiFe based cell chip (less than 1USD per one) in this work is autoclavable and can be used repeatedly. Another advantage of this cell chip is, if we add the number of test array, the sensitivity of the testing will be continueously increased. It will be carried out in our future work. However, FCM test can cost 90 USD or so per hour. Fourthly, this one step detection is fast. The preparation of the samples requires less procedures and the test itself is real-time.

Clinically, it is promising to be used for fast diagnosis, especially for fast screening test between patients' samples and standard controls. Therefore, only suspicious samples after our chip screening are sent for further quantitative test, such as FCM test.

Compared with the former reports of magnetic-based biomedical detection [12], we have updated it from specific detection for one type of cancer cell line into multiple cell lines detection. Besides, we managed integrated co-culture cell chip and set up one-step detection upon the chip. The integrated detection unit can co-work as a cell culture chip. Each detection unit integrated in the chip is also functional as our previous report [12]. Furthermore, it is also possible to achieve sensitive detection by the composition of the internal units, thus one cell cultural chip may be divided into several detection arrays for different tests. We also will improve their application in near future.

\section{Conclusion}

In summary, we successfully established a quick, sensitive method for testing cell surface receptor integrin based on a specially designed NiFe-based cell cul- 
tural biochip combined with functionalized magnetic nanoparticles. Compared with previous reported methods, our method has obvious advantages as follows: (1) When combined cell culture with detection, we shorten the test duration and ease the test procedure. Samples are almost ready for test after seeded on the chip and we saved single test for hours; the test is also real-time. (2) We improve the sensibility of the detection by combine the previous test elements into test array. (3) By this manner, the cost of qualitative comparison test of cell surface markers decreased.

\section{Acknowledgements}

This work is supported by National Key Basic Research Program (973 Project) (No. 2010CB933901 and 2011CB933100), National 863 Hi-tech Project of China (No. 2012AA022703), National Natural Scientific Fund (No. 81225010, 81101169 and 31100717), Shanghai Nanoproject (13NM1401500), Specialized Research Fund for the Doctoral Program of Higher Education (No. 20110073120072).

\section{References}

[1] C. Gui, X. Dai and D. Cui, "Advances of nanotechnology applied to biosensors", Nano Biomed. Eng. 3(4), 260-273 (2011). http://dx.doi.org/10.5101/ nbe.v3i4.p260-273

[2] J. Chen, D. Chen, T. Yuan and X. Chen, "Microfluidic PCR Chips", Nano Biomed. Eng. 3 (4), 203210 (2011). http://dx.doi.org/10.5101/nbe.v3i4. p203-210

[3] R. Vijayakumar, S. Jagannathan, P. R. Gandhi and S. Chaansha, "Nanorobotics: a newer platform for molecular diagnose", Nano Biomed. Eng. 3(3), 192201 (2011). http://dx.doi.org/10.5101/nbe.v3i3. p192-201

[4] X. Zhang, L. Jiang, C. Zhang, D. Li, C. Wang, F. Gao and D. Cui, "A silicon dioxide modified magnetic nanoparticles labeled lateral flow strips for HBs antigen", J. Biomed. Nanotechnol. 7(6), 776-781 (2011). http://dx.doi.org/10.1166/jbn.2011.1352

[5] R. Bashir, "BioMEMS: state-of-the-art in detection, opportunities and prospects", Adv. Drug Deliv. Rev. 56(11), 1565-1586 (2004). http://dx.doi.org/10. 1016/j.addr. 2004.03.002.

[6] D. R. Baselt, G. U. Lee, M. Natesan, S. W. Metzger, P. E. Sheehan and R. J. Colton, "A biosensor based on magnetoresistance technology", Biosens. Bioelectron. 13(7), 731-739 (1998). http://dx.doi.org/10.1016/ S0956-5663(98) 00037-2

[7] G. Kurlyandskaya and V. Levit, "Magnetic Dynabeads detection by sensitive element based on giant magnetoimpedance", Biosens. Bioelectron. 20(8), 16111616 (2005). http://dx.doi.org/10.1016/j.bios. 2004.04 .027
[8] S. Baranov, "Magnetic models of cast amorphous microwires", Surf. Eng. Appl. Electrochem. 47(4), 308-322 (2011). http://dx.doi.org/10.3103/ $\mathrm{S} 1068375511040028$

[9] F. Blanc-Beguin, S. Nabily, J. Gieraltowski, A. Turzo, S. Querellou and P. Salaun, "Cytotoxicity and GMI bio-sensor detection of maghemite nanoparticles internalized into cells", J. Magn. Magn. Mater. 321(3), 192-197 (2009). http://dx.doi.org/10.1016/ j.jmmm. 2008.08.104

[10] H. Yang, L. Chen, C. Lei, J. Zhang, D. Li, Z. M. Zhou, C. C. Bao, H. Y. Hu, X. Chen and F. Cui, "Giant magnetoimpedance-based microchannel system for quick and parallel genotyping of human papilloma virus type 16/18", Appl. Phys. Lett. 97 (4), 043702-043702-3 (2010). http://dx.doi.org/10. $1063 / 1.3467833$

[11] X. Zhi, Q. Liu, X. Zhang, Y. Zhang, J. Feng and D. Cui, "Quick genotyping detection of $\mathrm{HBV}$ by giant magnetoresistive biochip combined with PCR and line probe assay", Lab Chip. 12(4), 741 (2012). http:// dx.doi.org/10.1039/c2lc20949g

[12] L. Chen, C.-C. Bao, H. Yang, D. Li, C. Lei, T. Wang, H.-Y. Hu, M. He, Y. Zhou and D.-X. Cui, "A prototype of giant magnetoimpedance-based biosensing system for targeted detection of gastric cancer cells", Biosens. Bioelectron. 26(7), 3246-3253 (2011). http://dx.doi. org/10.1016/j.bios.2010.12.034

[13] G. Kurlyandskaya, "Giant magnetoimpedance for biosensing: Advantages and shortcomings", J. Magn. Magn. Mater. 321(7), 659-662 (2009). http://dx.doi. org/10.1016/j.jmmm. 2008.11.019

[14] L. Chen, Y. Zhou, C. Lei, Z. Zhou and W. Ding, "Giant magnetoimpedance effect in sputtered single layered $\mathrm{NiFe}$ film and meander $\mathrm{NiFe} / \mathrm{Cu} / \mathrm{NiFe}$ film", J. Magn. Magn. Mater. 322(19), 2834-2839 (2010). http://dx. doi.org/10.1016/j.jmmm.2010.04.038

[15] S. Petersen, J. M. Alonso, A. Specht, P. Duodu, M. Goeldner and A. delCampo, "Phototriggering of Cell Adhesion by Caged Cyclic RGD Peptides", Angew. Chem. Int. Ed. 47(17), 3192-3195 (2008). http://dx. doi.org/10.1002/anie. 200704857

[16] X. Chen, "Multimodality imaging of tumor integrin alphavbeta3 expression", Mini-Rev. Med. Chem. 6(2), 227 (2006). http://dx.doi.org/10. 2174/138955706775475975

[17] H. D. Han, L. S. Mangala, J. W. Lee, M. M. K. Shahzad, H. S. Kim, D. Shen, E. J. Nam, E. M. Mora, R. L. Stone, C. Lu, S. J. Lee, J. W. Roh, A. M. Nick, G. Lopez-Berestein and A. K. Sood, "Targeted Gene Silencing Using RGD-Labeled Chitosan Nanoparticles", Clin. Cancer Res. 16(15), 3910-3922 (2010). http:// dx.doi .org/10.1158/1078-0432. ccr-10-0005.

[18] X. Montet, K. Montet-Abou, F. Reynolds, R. Weissleder and L. Josephson, "Nanoparticle imaging of integrins on tumor cells", Neoplasia (New York, NY). 8(3), 214 (2006). http://dx.doi.org/10.1593/ neo. 05769

[19] Z. Li, P. Huang, X. Zhang, J. Lin, S. Yang, B. Liu, F. Gao, P. Xi, Q. Ren and D. Cui, "RGD- 
conjugated dendrimer-modified gold nanorods for in vivo tumor targeting and photothermal therapy", Mol. Pharm. 7(1), 94-104 (2009). http://dx.doi.org/10. $1021 / \mathrm{mp} 9001415$

[20] Y. Ye, S. Bloch, B. Xu and S. Achilefu, "Design, synthesis, and evaluation of near infrared fluorescent multimeric RGD peptides for targeting tumors", J. Med. Chem. 49(7), 2268-2275 (2006). http://dx.doi.org/ 10.1021/jm050947h

[21] I. Schmid, C. H. Uittenbogaart, B. Keld and J. V. Giorgi, "A rapid method for measuring apoptosis and dual-color immunofluorescence by single laser flow cytometry", J. Immunol. Methods. 170(2), 145 (1994). http://dx.doi.org/10.1016/ 0022-1759 (94) 90390-5

[22] C. Bao, N. Beziere, P. del Pino, B. Pelaz, G. Estrada, F. Tian, V. Ntziachristos, J. M. de la Fuente and D. Cui, "Gold nanoprisms as optoacoustic signal nanoamplifiers for in vivo bioimaging of gastrointestinal cancers", Small 9(1), 68-74 (2013). http://dx.doi.org/ 10.1002/smll.201201779

[23] H. Hu, H. Yang, D. Li, K. Wang, J. Ruan, X. Zhang, J. Chen, C. Bao, J. Ji and D. Shi, "The potential of magnetic nanocluster and dual-functional protein-based strategy for noninvasive detection of HBV surface antibodies", Analyst. 136(4), 679-683 (2010). http://dx. doi.org/10.1039/c0an00517g

[24] B. Pan, D. Cui, Y. Sheng, C. Ozkan, F. Gao, R. He, Q. Li, P. Xu and T. Huang, "Dendrimer-modified magnetic nanoparticles enhance efficiency of gene delivery system", Cancer research. 67(17), 8156-8163 (2007). http://dx.doi.org/10. 1158/0008-5472. CAN-06-4762

[25] G. Gao, P. Huang, Y. Zhang, K. Wang, W. Qin and D. Cui, "Gram scale synthesis of superparamagnetic $\mathrm{Fe}_{3} \mathrm{O}_{4}$ nanoparticles and fluid via a facile solvothermal route", Crystengcomm. 13(6), 1782-1785 (2011). http://dx.doi.org/10.1039/c0ce00584c
[26] G. Gao, P. Huang, K. Wang, R. He and D. Cui, "Gramscale synthesis and shape evolution of micro- $\mathrm{CaCO}_{3}$ ", Powder Technol. 205(1), 270-275 (2011). http://dx. doi.org/10.1016/j.powtec. 2010.09.032

[27] H. Hu, H. Yang, P. Huang, D. Cui, Y. Peng, J. Zhang, F. Lu, J. Lian and D. Shi, "Unique role of ionic liquid in microwave-assisted synthesis of monodisperse magnetite nanoparticles", Chem. Commun. 46(22), 38663868 (2010). http://dx.doi.org/10.1039/b927321b

[28] C. Zhang, M. Jugold, E. C. Woenne, T. Lammers, B. Morgenstern, M. M. Mueller, H. Zentgraf, M. Bock, M. Eisenhut and W. Semmler, "Specific targeting of tumor angiogenesis by RGD-conjugated ultrasmall superparamagnetic iron oxide particles using a clinical 1.5-T magnetic resonance scanner", Cancer Res. 67(4), 1555-1562 (2007). http://dx.doi.org/10. 1158/0008-5472. CAN-06-1668

[29] G. Thumshirn, U. Hersel, S. L. Goodman and H. Kessler, "Multimeric cyclic RGD peptides as potential tools for tumor targeting: solid-phase peptide synthesis and chemoselective oxime ligation", Chem. Eur. J. 9(12), 2717-2725 (2003). http://dx.doi.org/10. 1002/chem. 200204304

[30] E. D. Hay, "Collagen and other matrix glycoproteins in embryogenesis", Cell biology of extracellular matrix 2, 419-462 (1991).

[31] J. Madri, "Endothelial cell-matrix interactions in hemostasis", Progress in hemostasis and thrombosis. 6, 1 (1982).

[32] U. Saarialho-Kere, S. Kovacs, A. Pentland, J. Olerud, H. Welgus and W. Parks, "Cell-matrix interactions modulate interstitial collagenase expression by human keratinocytes actively involved in wound healing", J. Clin. Invest. 92(6), 2858-2866 (1993). http://dx.doi. org/10.1172/JCI116906

[33] G. G. Vaday and O. Lider, "Extracellular matrix moieties, cytokines, and enzymes: dynamic effects on immune cell behavior and inflammation", J. Leukoc. Biol. 67(2), 149-159 (2000). 